\title{
Immune Response To SARS-Cov-2 In Severe And Long COVID-19
}

\section{Tomonari Sumi ( $\nabla$ sumi@okayama-u.ac.jp )}

Okayama University

\section{Kouji Harada}

Toyohashi University of Technology

\section{Research Article}

Keywords:

Posted Date: February 17th, 2022

DOI: https://doi.org/10.21203/rs.3.rs-1348061/v1

License: (a) (i) This work is licensed under a Creative Commons Attribution 4.0 International License. Read Full License 


\title{
Immune response to SARS-CoV-2 in severe and long COVID-19
}

\author{
Tomonari Sumi ${ }^{1,2, *}$ and Kouji Harada ${ }^{3,4}$
}

${ }^{1}$ Research Institute for Interdisciplinary Science, Okayama University, 3-1-1 Tsushima-Naka, Kita-ku, Okayama 700-8530, Japan

2Department of Chemistry, Faculty of Science, Okayama University, 3-1-1 Tsushima-Naka, Kita-ku, Okayama 700-8530, Japan

${ }^{3}$ Department of Computer Science and Engineering, Toyohashi University of Technology, Tempaku-cho, Toyohashi 441-8580, Japan

${ }^{4}$ Center for IT-Based Education, Toyohashi University of Technology, Tempaku-cho, Toyohashi, Aichi, 441-8580, Japan

*sumi@okayama-u.ac.jp

\begin{abstract}
(max. 150 words)
COVID-19 is mild to moderate in otherwise healthy individuals but may nonetheless cause life-threatening disease and/or a wide range of persistent symptoms. The general determinant of disease severity is age mainly because the immune response declines in aging patients. Here, we developed a mathematical model of the immune response to SARS-CoV-2 and revealed that typical age-related risk factors such as only a several $10 \%$ decrease in innate immune cell activity and inhibition of type-I interferon signaling by autoantibodies drastically increased the viral load. It was reported that the numbers of certain dendritic cell subsets remained less than half those in healthy donors even seven months after infection. Hence, the inflammatory response was ongoing. Our model predicted the persistent DC reduction and showed that certain patients with severe and even mild symptoms could not effectively eliminate the virus and could potentially develop long COVID.
\end{abstract}




\section{Introduction}

The emergence of severe acute respiratory syndrome coronavirus 2 (SARS-CoV-2) has caused an unprecedented ongoing global pandemic known as coronavirus 2019 (COVID-19) ${ }^{1,2}$. The disease has heterogeneous characteristics. It may be asymptomatic, induce mild symptoms, or cause critical illness. In the latter case, $10-20 \%$ of all symptomatic patients are at elevated risks of multiple organ system involvement and mortality ${ }^{1,3,4}$. There is limited experimental or clinical evidence that the virus per se is mainly responsible for the heterogeneity of the disease it causes. In contrast, there is growing evidence that the host accounts for the observed variability in disease severity, infection rate, and long-term disease symptoms ${ }^{5}$. Therefore, better understanding of the innate and adaptive immune response in mild and potentially fatal COVID-19 is crucial for developing diagnostic markers and therapeutic strategies.

Quantitative methods have been developed to analyze the dynamics of SARS-CoV-2 infection within the host ${ }^{6-11}$. The present study proposes a mathematical model for the immune response to SARS-CoV-2 (Fig. 1). It reveals the roles of innate and adaptive immunity and examines the mechanisms of the development of severe COVID-19 in response to age-related risk factors. SARS-CoV-2 has already mutated to evade the immune response. For example, it dysregulates type-I interferon (IFN1) which is a cytokine secreted by infected host cells ${ }^{12}$. Here, model simulations were used to assess age-related risk factors ${ }^{13}$, e.g., virally-mediated suppression effect of IFN1 production by infected epithelial cells and the influences of IFN1-neutralizing autoantibodies.

Dendritic cells (DCs) are key orchestrators of the immune response. However, their functionality declines with age ${ }^{14-16}$. In contrast, aging does not substantially influence DC abundance or phenotype. Nevertheless, the capacity of DCs to phagocytose antigens and migrate is impaired with age ${ }^{14,15}$. In addition, the ability of DCs to present antigens to CD4 and CD8 T cells also decreases during the aging process ${ }^{16}$. The model simulations also demonstrated the impact of age-associated DC impairment on COVID-19 severity.

Influenza A virus infection generally causes self-limited bronchitis and possibly severe pneumonia ${ }^{17}$. In contrast, SARS-CoV-2 can infect a wide range of human cell types as the symptoms of COVID-19 involve several different organ systems. The spike subunit of SARS-CoV-2 binds human angiotensin-converting enzyme 2 receptor (ACE2) and is primed by the cellular serine proteases TMPRSS2 and TMPRSS4 so that the virus can enter other host cells ${ }^{18}$. ACE2 is expressed in the entire human respiratory system, brain endothelium, and vascular smooth muscle cells ${ }^{19,20}$. Moreover, ACE2 and TMPRSS2 are expressed in esophageal keratinocytes, renal proximal tubules, pancreatic $\beta$-cells, and gastrointestinal epithelial cells ${ }^{4,20-22}$. These facts are consistent with the observation that in certain post-acute sequelae of COVID-19 patients, SARS-CoV-2 maintains chronic symptoms by persisting in certain sites or tissue reservoirs after acute infection ${ }^{23}$. Possibly related to 
those, the numbers of CD1c+ myeloid and plasmacytoid DCs remained low even seven months after SARS-CoV-2 infection whether or not the patients were previously hospitalized ${ }^{24}$. Our model reproduced long-term DC count reduction and showed that ongoing DC-induced inflammation was attributed to persistent viral infection that the host could not remove. The model simulations also predicted that successful elimination of the virus depends on the capacity of the host immune response which is directly related to viral load.

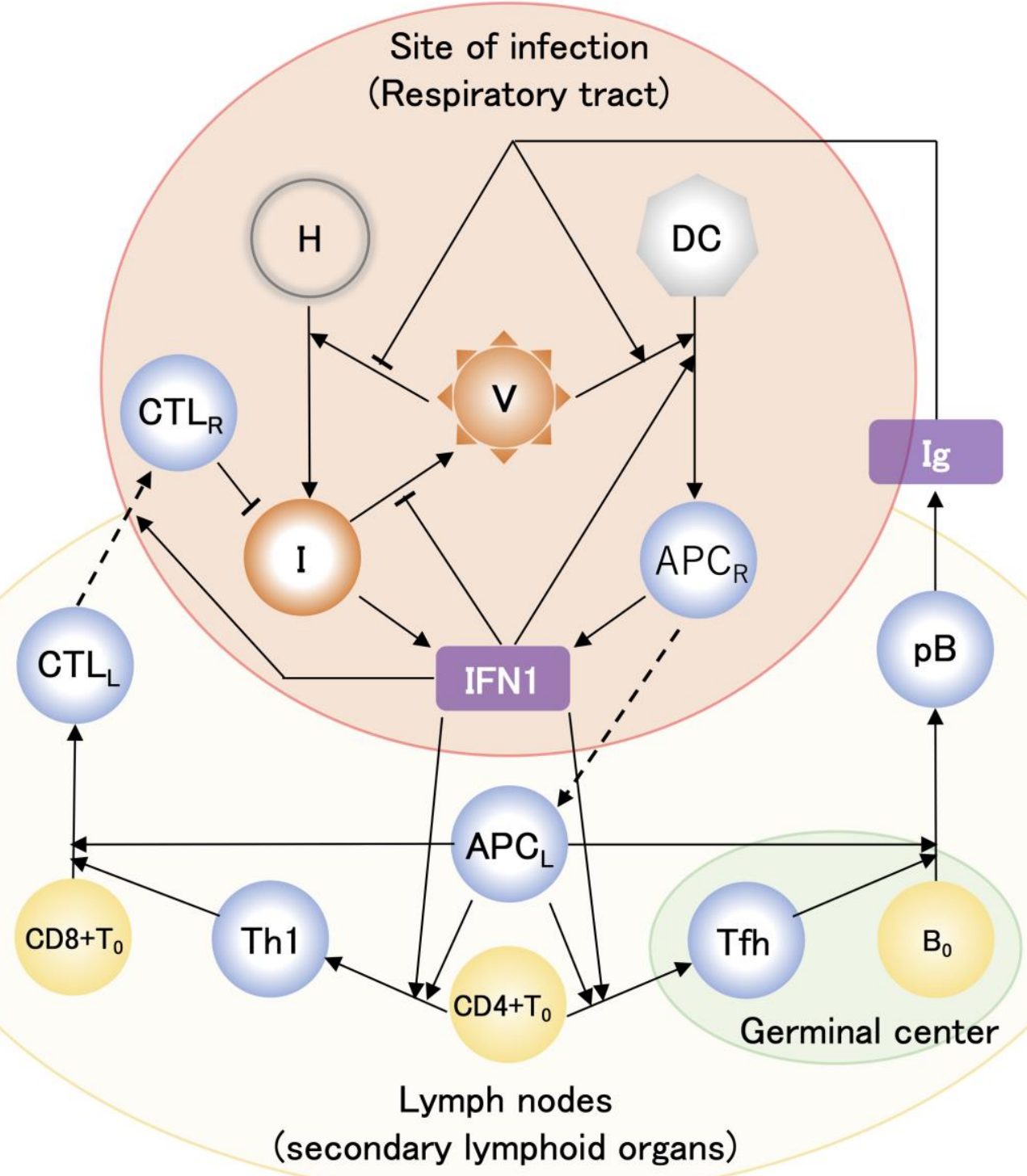

Figure 1. Mathematical model of host immune response to SARS-CoV-2 infection. Solid arrow denotes either activation or differentiation. Dashed and blunt arrows denote migration and inhibition, respectively. Model variables include target healthy epithelial cells [H], infected cells [I], viral loads [V], dendritic cells [DC], antigen-presenting cells generated from DC at infection sites (respiratory tract) $\left[\mathrm{APC}_{\mathrm{R}}\right]$ and in lymph nodes $\left[\mathrm{APC}_{\mathrm{L}}\right]$, naïve $\mathrm{CD} 4+$ and $\mathrm{CD} 8+\mathrm{T}$ cells, $\left[\mathrm{CD} 4+\mathrm{T}_{0}\right]$ and $\left[\mathrm{CD} 8+\mathrm{T}_{0}\right]$, naïve $\mathrm{B}$ cells $\left[\mathrm{B}_{0}\right]$, type-I helper T cells $[\mathrm{Th} 1]$, $\mathrm{T}$ follicular helper cells [Tfh], cytotoxic T lymphocytes in lymph nodes [CTL $\mathrm{L}$ ] and infection sites $\left[\mathrm{CTL}_{\mathrm{R}}\right]$, 
plasma B cells [pB], type-I interferon [IFN1], and immunoglobulin [Ig].

\section{Results}

\section{Baseline model solution for immune response to SARS-CoV-2 infection}

Ordinary differential equations 1-16 were solved using the initial values of the variables (Table S1, Supplementary Information (SI)) and the parameters provided in Table S2 (SI). Figure 2 shows the variables for the baseline model solution plotted as a function of the number of days after infection. The viral load $[\mathrm{V}]$ was compared against the model solution that was determined by fitting to the available viral load data ${ }^{8}$ along with the data for Singapore COVID-19 patients ${ }^{9,25}$ (Fig. 2a). The time to the onset of symptoms after infection $(\sim 5.62 \mathrm{~d})$ and the error $( \pm 0.48 \mathrm{~d})$ were mathematically determined using viral load data ${ }^{9}$ are also represented by the vertical lines in Fig. 2a. The peak viral load was attained at $\sim 2 \mathrm{~d}$ after symptom onset as previously reported ${ }^{8}$ and is represented by the blue line. Figure $2 b$ shows that the immunoglobulin concentration $[\operatorname{Ig}]$ time course was consistent with experimental data. Pre-existing Ig was initially consumed and the [Ig] increased thereafter in response to plasma B cell activation and Ig secretion. The correlation between infected cell [I] and infected cell $[\mathrm{V}]$ is shown in Fig. 2c. The observed steep rise in [V] immediately after infection was attributed to the strong infectivity of SARS-CoV-2. After [V] declined to a minimum, it gradually increased towards its steady state as [I] also increased. The validity of our prediction regarding persistent SARS-CoV-2 infection will be addressed in terms of longitudinal observations of the DC immune response ${ }^{24}$ (Fig. 3) related to long COVID or post-acute sequelae of COVID-19 (PASC) ${ }^{23}$.

Figure $2 \mathrm{~d}$ shows the time course for the transmission of innate and acquired immunocyte activation. $\mathrm{DC}$ transformation into antigen-presenting cells $\left(\mathrm{APC}_{\mathrm{R}}\right)$ at the infection sites begins at the onset of the viral infection and rapidly increasing after symptom onset (vertical blue lines). $\mathrm{APC}_{\mathrm{R}}$ migrates from the infection site to a lymph node (Fig. 1) and $\mathrm{APC}_{\mathrm{L}}$ increases (Fig. 2d). $\mathrm{APC}_{\mathrm{L}}$ induces naïve CD4+ T cells to differentiate into type-I helper T cells (Th1) and T follicular helper cells (Tfh). Thence, $\mathrm{APC}_{\mathrm{L}}$ and $\mathrm{Th} 1$ induce naïve $\mathrm{CD} 8+\mathrm{T}$ cells $\left(\mathrm{CD} 8+\mathrm{T}_{0}\right)$ to differentiate into cytotoxic $\mathrm{T}$ lymphocytes $\left(C T L_{L}\right)$. From there, the $C T L_{L}$ migrate to the infection sites (Fig. 1) and [CTL $L_{R}$ increases. Similarly, $\mathrm{APC}_{\mathrm{L}}$ and $\mathrm{Tfh}$ induces naïve $\mathrm{B}$ cells $\left(\mathrm{B}_{0}\right)$ to differentiate into plasma B cells (pB) (Fig. 1) and $[\mathrm{pB}]$ increases.

Figures $2 \mathrm{e}$ and $2 \mathrm{f}$ show the dominant fluxes that explain the observed variations in $[\mathrm{I}]$ and [V], respectively. Viral infection in healthy epithelial cells $[\mathrm{H}]$ increases [I]. Thereafter, $\mathrm{CTL}_{\mathrm{R}}$ kill I and cause $[\mathrm{I}]$ to decline sharply (Fig. 2e). The number of viral particles that have been replicated by infected cells within the host is reduced by natural viral degradation. However, this process is comparatively slow (Fig. 2f). Figure $2 \mathrm{~g}$ shows the time course for $[\mathrm{I}]$ and $\left[\mathrm{APC}_{\mathrm{R}}\right]$ involved in IFN1 production. $[\mathrm{I}]$ increased faster than $\left[\mathrm{APC}_{\mathrm{R}}\right]$ whereas the increases and decreases in [IFN1] were 
synchronized with the changes in $\left[\mathrm{APC}_{\mathrm{R}}\right]$ rather than $[\mathrm{I}]$. In fact, the IFN1 production rate used for the baseline model was 1,000-fold lower in infected cells than $\mathrm{APC}_{\mathrm{R}}$ (Table S2, SI). This is because SARS-CoV-2 possesses several mechanisms to evade the IFN1-mediated immune response ${ }^{12}$. However, if a virus lacked these mechanisms, it can be more rapidly cleared from the host as shown by the blue solid line in Fig. $2 \mathrm{~h}$.
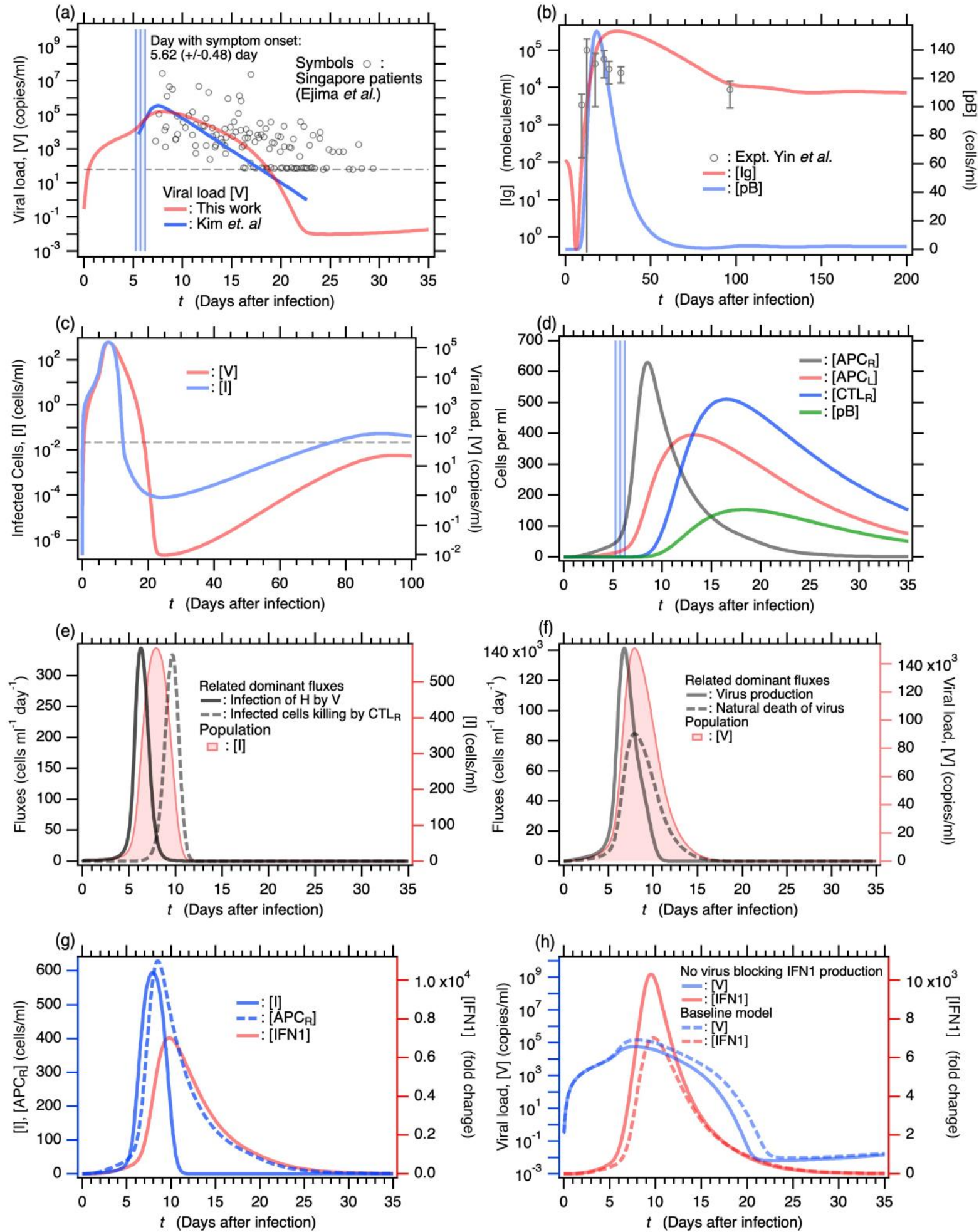

Figure 2. Baseline model solution for immune response as function of number of days after SARS-CoV-2 
infection. (a) Comparison of baseline model solution for viral load [V] calculated from our mathematical model against $[\mathrm{V}]$ that Kim et al. determined by fitting a target cell-limited model to viral load data ${ }^{8}$. Symbols are viral load data for Singapore COVID-19 patients ${ }^{9,25}$. Vertical blue lines show days of symptom onset and error estimated by mathematical method of viral load data ${ }^{9}$. Dashed horizontal line indicates viral detection limit. (b) Comparison of $[\mathrm{Ig}]$ obtained against longitudinally observed clinical data ${ }^{26}$. Plasma B cell concentration [pB] is also shown (right axis). (c) Long-term time course of infected cell [I] concentration (left axis) and [V] (right axis). Dashed horizontal line is same as that in (a). (d) Time courses of concentrations of immunocytes [APC $C_{R}$, $\left[\mathrm{APC}_{\mathrm{L}}\right],\left[\mathrm{CTL}_{\mathrm{R}}\right]$, and $[\mathrm{pB}]$. Vertical blue lines are same as those in (a). (e) Fluxes contributing to variation in [I], namely, viral $\mathrm{H}$ infection and I killing by cytotoxic T lymphocytes (left axis). For comparison, [I] time course is also shown (right axis). (f) Fluxes contributing to variation in [V], namely, virus production by I and natural V degradation, are shown (left axis). Time course of [V] is also shown (right axis). (g) Time courses of [I] and $\left[\mathrm{APC}_{\mathrm{R}}\right]$ are shown (left axis). Both secrete IFN1 (right axis). (h) Time courses of [V] (left axis) and [IFN1] (right axis) for model solution without mechanisms of evading IFN1 secretion are compared against those of baseline model (broken lines).

\section{Dendritic cell deficiency persists for $>7$ mo after SARS-CoV-2 infection}

DCs play key roles in defending against viral infections. DCs that capture viruses function as APCs while plasmacytoid DCs (pDCs) produce abundant IFN1. Whether or not COVID-19 patients were previously hospitalized, the numbers of their CD1c+ myeloid DCs and pDCs were lower than those for healthy donors during the acute infection phase and even 7 mo after the initial SARS-CoV-2 infection (Fig. 3a) ${ }^{24}$. Hence, the DCs induce and partially sustain ongoing inflammation that can cause long COVID or PASC. Our baseline simulation predicted that the number of DCs rapidly decreases during the acute phase and increases thereafter but is nonetheless lower than it was before the infection (Fig. 3b). The simulated proportions of DC reduction in infected patients compared with healthy donors were consistent with those determined by clinical observation (Fig. 3a) ${ }^{24}$. Figure $2 \mathrm{c}$ shows that [V] increases after decreasing to a minimum possibly because certain viruses cannot be removed by the host as a consequence of persistent infection. In our baseline simulation, then, long-term DC deficiency was attributable to persistent SARS-CoV-2 infection. Nevertheless, persistent DC deficiency was commonly observed for both short-term and long-term COVID-19 symptoms ${ }^{24}$. 

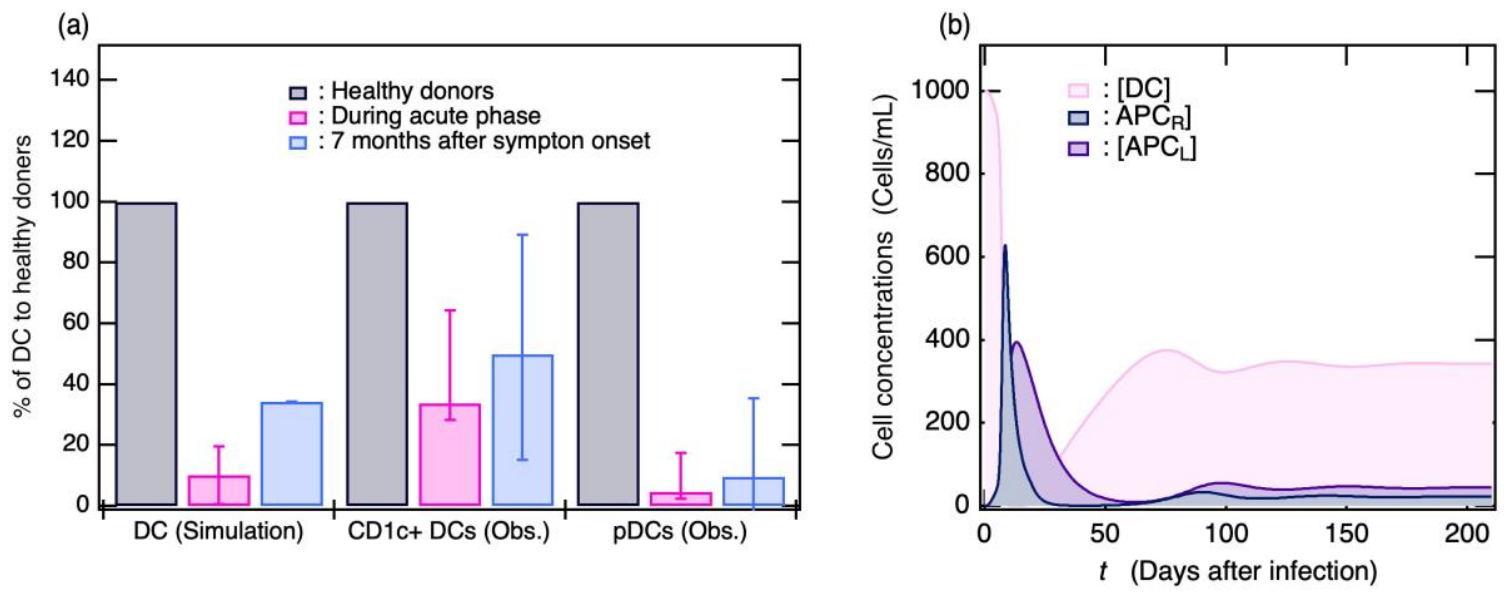

Figure 3. Substantial decrease in DC level in response to SARS-CoV-2 infection. (a) Bar graphs represent \% DC during acute infection phase and 7 mo after symptom onset compared with \% DC in healthy donors. Clinical observation data were derived from literature ${ }^{24}$. (b) Time courses of $[\mathrm{DC}],\left[\mathrm{APC}_{\mathrm{R}}\right]$, and $\left[\mathrm{APC}_{\mathrm{L}}\right]$ obtained from simulation. In (a), average simulated DC values were taken $3 \mathrm{~d}$ and $14 \mathrm{~d}$ after symptom onset in same manner as clinical trials ${ }^{24}$. For clinical data, \% DC at 7 mo after symptom onset was average for non-hospitalized and previously hospitalized patients.

\section{Deficient immune responses might cause severe COVID-19}

Attenuation of the immune system during aging is associated with increased susceptibility to various infectious diseases, a decrease in the ability to fight new infections, re-emergence of latent infections, and increases in disease severity. Older people are at a much higher risk of developing severe or fatal COVID-19 than younger people ${ }^{27}$. We examined the effects of aging on the immune system to understand the risk factors and typical mechanisms related to COVID-19 exacerbation. There were no major differences between young and elderly subjects in terms of the numbers or phenotypes of their DC subsets. In contrast, the ability of DCs to phagocytose antigens, migrate, and prime $\mathrm{T}$ cell responses declined with advancing age ${ }^{14,16}$. Thus, we explored how reductions in DC transformation into APC, APC migration towards lymph nodes, and CD4+ $\mathrm{T}_{0}$ differentiation into Th1 and Tfh (Table S4, SI) affect COVID-19 severity.

Congenital and acquired defects in IFN1 signaling can result in severe COVID-19. IFN1 autoantibodies were detected in plasma samples from a large cohort comprising COVID-19 patients and pre-pandemic controls ${ }^{13}$. The incidence of IFN1-neutralizing autoantibodies increased especially in subjects $>70 \mathrm{y}$ of age in the control cohort. Hence, the autoantibodies targeting IFN1 manifested a common form of acquired immunodeficiency associated with $\sim 20 \%$ of all COVID-19 fatalities ${ }^{13}$. In the present study, we investigated how reductions in IFN1 signaling affects the suppression of viral replication, the migration of $\mathrm{APC}_{\mathrm{R}}$ and $\mathrm{CTL}_{\mathrm{L}}$, the differentiation of $\mathrm{CD} 4+\mathrm{T}_{0}$ into Th1 and $\mathrm{Tfh}$ (Table S4, SI), and consequently the severity of COVID-19. With advancing age, the number of naïve CD8+ 
$\mathrm{T}$ cell decreases by approximately one order of magnitude whereas the numbers of naïve CD4+ T and $\mathrm{B}$ cells do not ${ }^{28}$. Thus, we also examined how age-related reductions in [CD8+ $\left.\mathrm{T}_{0}\right]$ (Table S4, SI) affect the viral load time course (Fig. 4).

Reduced APC activity and deficient IFN1 signaling decrease [V] immediately after symptom onset and also contribute to higher long-term viral loads (Fig. 4a). The increase in [V] following symptom onset is related to an increase in [I] (Fig. 4b). In contrast, a decrease in [CD8+ $\left.\mathrm{T}_{0}\right]$ by one order of magnitude does not affect [V] immediately after symptom onset but does retard virus removal (Fig. 4a). In this case, the lack of any increase in the maximum [V] is attributed mainly to the lack of increase in the maximum [I] (Fig. 4b). Prolonged infection caused by a dramatic reduction in [CTL ${ }_{R}$ ] might account for the observed delays in viral clearance despite moderate maximum viral loads (Figs. $4 \mathrm{~b}$ and $4 \mathrm{e}$, red line). As the decrease in [CD8+ $\left.\mathrm{T}_{0}\right]$ affects neither [Th1] nor [Tfh], it was omitted from Figs. 4c and 4d. Impairment of APC activity and IFN1 signaling ordinarily affects naïve CD4+ T cell priming into Th1 and Tfh (Figs. 4c and 4d), thereby lowering [CTLR] and [Ig] (Figs. 4e and 4f). In response to deficient APC activity and neutralization of IFN1 signaling, maximum [I] is higher for the former than the latter, maximum [V] is higher for the latter than the former, and both conditions do not significantly differ in terms of their effects on maximum $\left[\mathrm{CTL}_{\mathrm{R}}\right]$ or $[\mathrm{Ig}]$. Therefore, IFN1 signaling profoundly affects the suppression of viral replication in infected cells. Taken together, the foregoing findings indicate that all patients that are partially deficient in innate and/or acquired immunity because of inflammation and disease are also potentially at high risk of severe or even fatal COVID-19. 

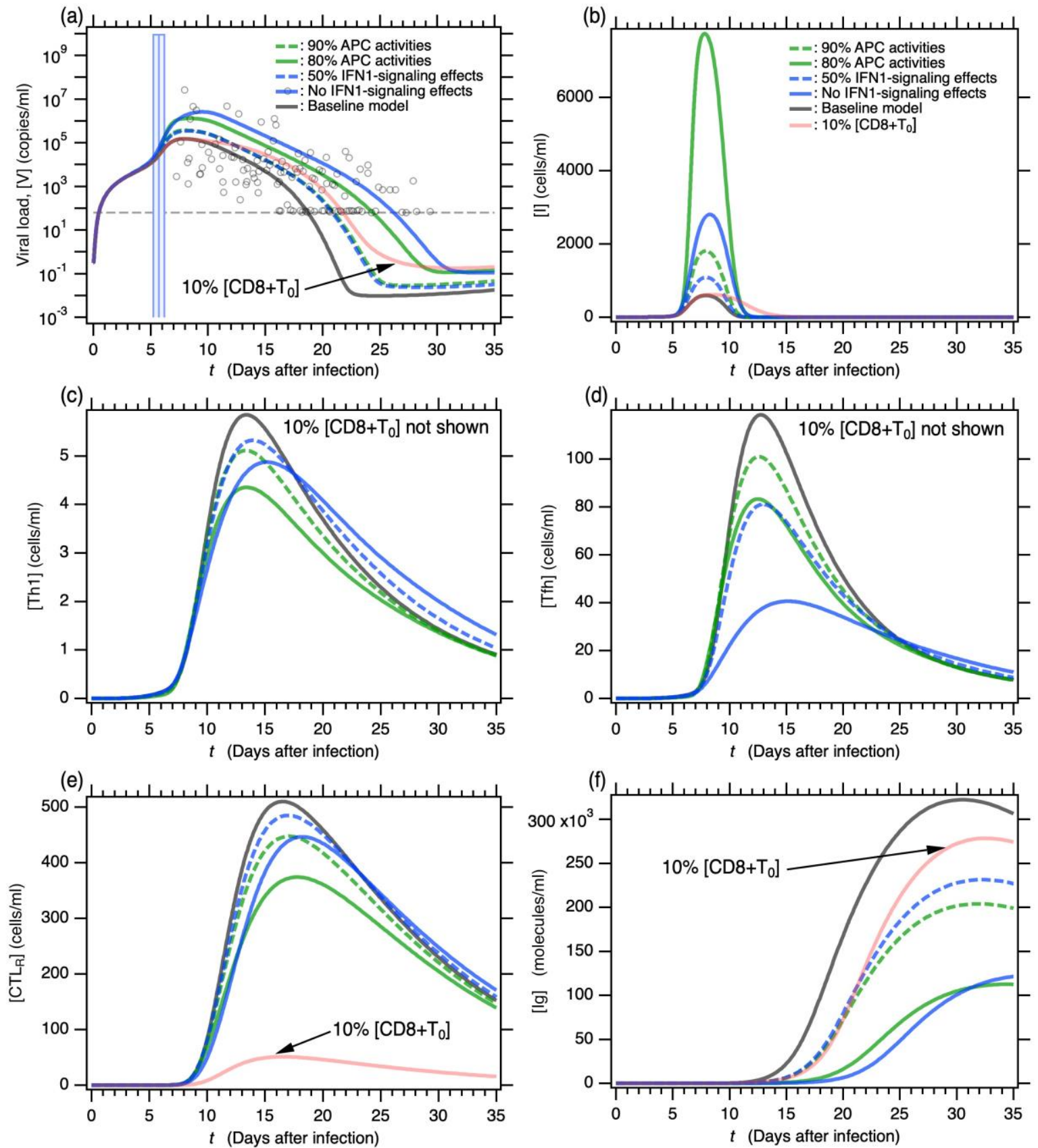

Figure 4. Model solutions for severe COVID-19 symptoms depending on typical age-related risk factors.

Time courses of (a) viral load [V], (b) infected cells [I], (c) type-I helper T cells [Th1], (d) T follicular helper cells $[\mathrm{Tfh}]$, (e) cytotoxic $\mathrm{T}$ lymphocytes at infection sites $\left[\mathrm{CTL}_{\mathrm{R}}\right]$, and (f) immunoglobulin $[\mathrm{Ig}]$ in patients with various age-related risk factors. Five age-related risk factors, 90\% APC activity (model 1), 80\% APC activity (model 2), 50\% reduction in IFN1 signaling (model 3), no IFN1 signaling (model 4), and 10\% [CD8+ $\left.\mathrm{T}_{0}\right]$ (model 5) were examined. Parameters used in these models are listed in Table S4 (SI). 


\section{The probability of complete SARS-CoV-2 elimination increases with the ability of the immune system to suppress viral replication}

The baseline simulation (Fig. 2) indicates that numerous patients cannot successfully remove SARS-CoV-2. The persistent viral infection within the host might cause long COVID or PASC. To evaluate this prediction, we investigated the relationships between [V] after infinite time (steady-state $[\mathrm{V}])$ and the model parameters. The model parameters significantly reducing the steady-state values of $[\mathrm{V}]$ and $[\mathrm{I}]$ were disclosed by a steady-state sensitivity analysis ${ }^{29}$. The most sensitive parameters were associated with Ig production $\left(\pi_{I g}, \pi_{p B}\right.$, and $\left.\pi_{T f h}\right)$ and APC activation $\left(\pi_{A P C}, \alpha_{A P C}\right.$, and $\left.\mu_{A P C}\right)$ except for the initial value of cell concentrations (Eqs. 1-16). The [V] time courses for six models with severalfold increases in the Ig production and/or APC activation parameters (Table S5, SI) are shown in Fig. 5a along with the baseline model. When $[\mathrm{V}]<10^{-4}$, time evolution discontinued and it was assumed that the virus was entirely eliminated from the host. Figure 5a shows that not only long-term $[\mathrm{V}]$ but also maximum $[\mathrm{V}]$ decreased with increasing parameter value. Hence, the maximum [V] decreased with the steady-state [V] (Fig. 5b). The [V] time courses in these models deviated from the clinical observation data (Fig. 2a). These findings suggest that patients cannot completely remove even average SARS-CoV-2 loads that are undergoing replication. As the steady-state $[\mathrm{V}]$ decreases, the minimum $[\mathrm{V}]$ becomes so small that it is effectively zero (Fig. 5c). In this case, the patient is virtually cured as [V] and [I] should stochastically converge to zero. Therefore, when Ig production and APC activity are sufficiently high, the patient is asymptomatic, viral replication is suppressed, the virus may have been successfully removed, and the patient is cured. Otherwise, viral infection remains in the host and can progress into long COVID or PASC. 


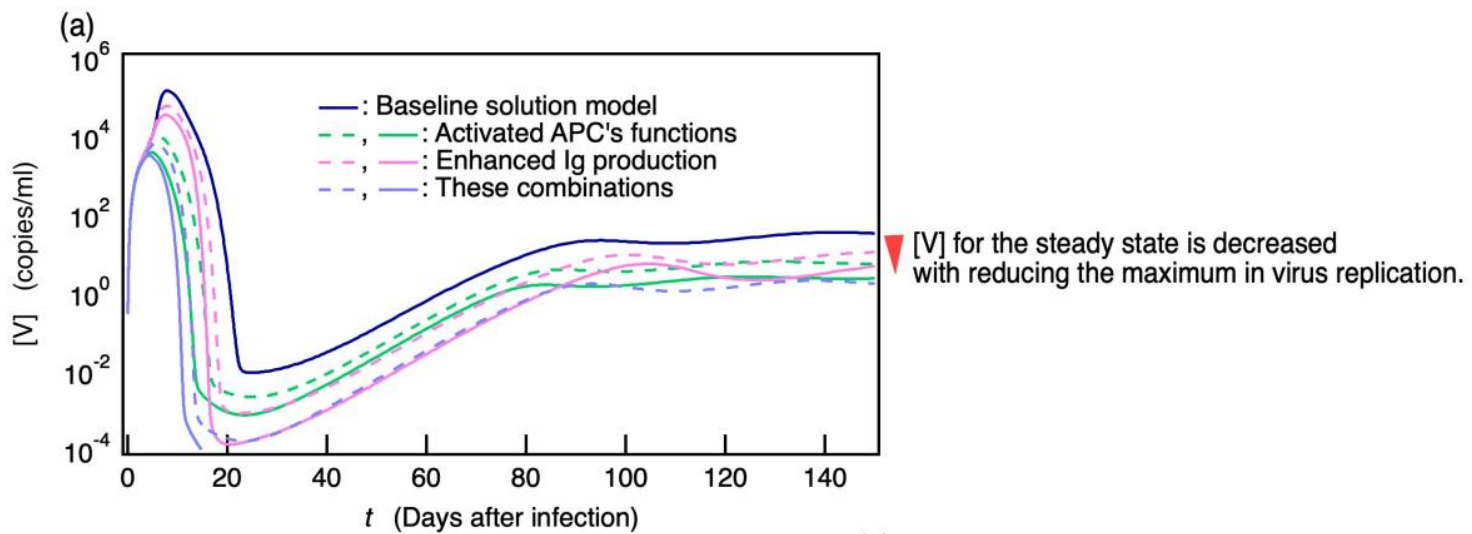

(b)

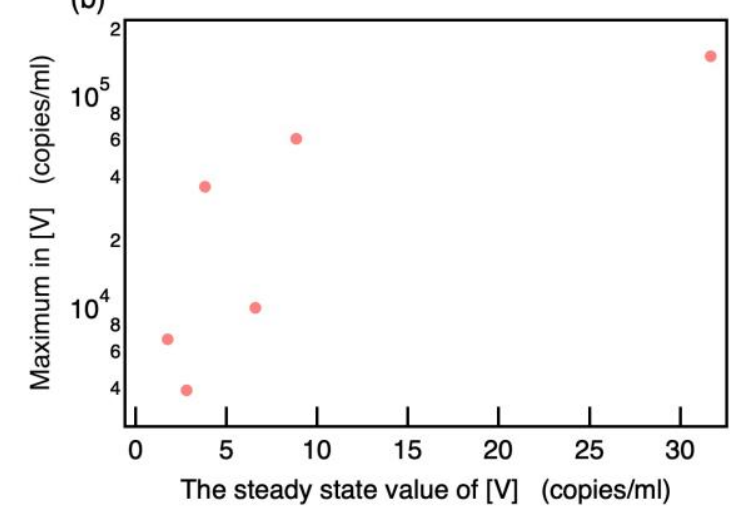

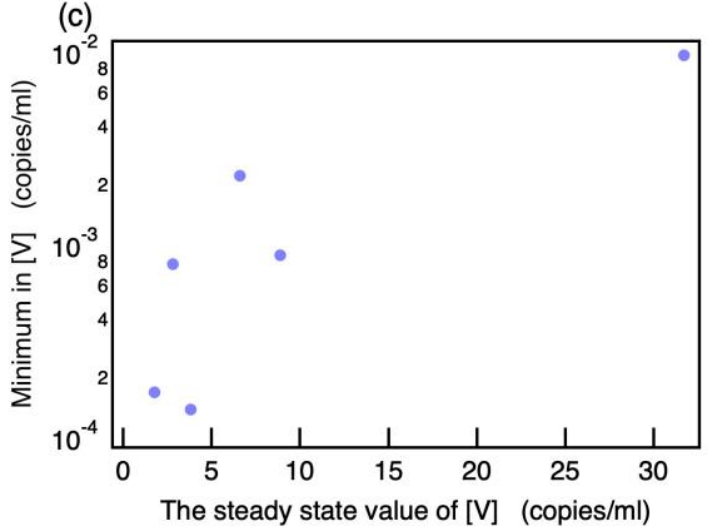

Figure 5. Ability of the immune response to suppress viral replication is necessary for complete

SARS-CoV-2 clearance. (a) Time courses of [V] in six models with severalfold increases in parameters related to APCs function and/or Ig production (Table S5, SI). (b) Maximum and (c) minimum [V] shown in (a) vs. steady-state [V]. In models of the highest immune capacity, viral load becomes very low ([V] $\left.<\sim 10^{-4}\right)$, virus is assumed to be completely eliminated from host, and time evolution is discontinued in (a). Hence, [V] for this scenario is not shown in (b) and (c). In (a), colored solid line represents stronger immune response than colored dashed line.

\section{Discussion}

SARS-CoV-2 can reach and infect the cells in multiple organs and tissues via hematogenous diffusion from heavily infected airways and lungs ${ }^{23}$. Influenza A virus causes a self-limited acute viral infection in the upper respiratory tract ${ }^{17}$. In contrast, ACE2 and TMPRSS2 enable SARS-CoV-2 to infect and penetrate a wide range of host cell types ${ }^{4,20-22}$. Evidence for systemic SARS-CoV-2 infection was provided from the complete autopsies of 44 patients with COVID-19 and demonstrated SARS-CoV-2 distribution, replication, and cell-type specificity throughout the human body. The virus was widely distributed even in deceased patients with asymptomatic to mild COVID-19 ${ }^{30}$. In fact, viral replication was detected in multiple extrapulmonary tissues and systemic infection persisted for more than several months.

PASC is being diagnosed in patients with severe acute COVID-19 as well as those with only 
mild or even no symptoms ${ }^{31}$. The long-term symptoms observed in PASC patients may be the consequences of organ and tissue injury caused by SARS-CoV-2 and/or coagulation and inflammation during acute COVID-19 ${ }^{32}$. In contrast, SARS-CoV-2 may remain within certain PASC patients, thereby causing chronic inflammation and dysfunction in certain organs and tissues. Several studies reported that patients infected with SARS-CoV-2 may not fully clear it for very long periods of time ${ }^{33-35}$. In a trial on 203 post-symptomatic participants with previous RT-PCR-verified SARS-CoV-2 infection, $5.3 \%$ of the subjects remained virus-positive even $90 \mathrm{~d}$ after recovery ${ }^{35}$. There were no differences between PCR-positive and PCR-negative subjects in terms of SARS-CoV-2-specific Ig. However, the PCR-positive group presented with significantly stronger SARS-CoV-2-specific CD8+T-cell responses ${ }^{35}$.

Dendritic cells (DCs) are components of innate immunity and play key roles in host SARS-CoV-2 response. Recovery of DC defects after COVID-19 is vital as normalization of the innate immune system after acute insults is required for appropriate responses to new microbial challenges. However, patients with acute SARS-CoV-2 infection present with substantially reduced DC counts that might not normalize even 7 mo after the initial acute SARS-CoV-2 infection ${ }^{24}$ (Fig. 3). The observed long-term decrease in DC number may be explained by the migration of these cells to inflammatory sites caused by persistent SARS-CoV-2 infection and contributing to long COVID.

Numerous studies have reported persistent single-strand RNA virus infections ${ }^{36-38}$ especially in the central nervous system (CNS) ${ }^{39}$. The CNS is considered a target for several different persistent viral infections as neurons are post-mitotic single cells that persist throughout the entire lifetime of the host. Thus, neurons may provide a more protective environment for long-term viral persistence than rapidly multiplying cells that can sequester microbial pathogens ${ }^{39}$. As a well-known case, hepatitis $\mathrm{C}$ virus (HCV) also establishes persistent infection by evading the host innate immune response ${ }^{40,41}$. Therefore, persistence of SARS-CoV-2 infection can be expected in patients with PASC symptoms. In an earlier clinical trial, however, only $\sim 5 \%$ of all subjects were positive for SARS-CoV-2 according to RT-PCR nasopharyngeal testing $\sim 90 \mathrm{~d}$ after infection and there was no apparent transmission to close contacts ${ }^{35}$. Nevertheless, there is no consensus that patients with persistent SARS-CoV-2 infection are not contagious. Persistent SARS-CoV-2 infection and infectivity merit further investigation so that treatments for PASC may be developed and the COVID-19 pandemic may be managed more effectively.

Our mathematical model of the host immune response to SARS-CoV-2 demonstrated that age-related risk factors such as a decrease in innate immune cell activity and/or an increase in autoantibody-mediated IFN1 signaling inhibition markedly increased viral load. Our model also predicted persistent reductions in DC abundance and showed that patients with severe and even mild symptoms may develop long COVID-19 as they may not effectively eliminate the virus. However, the 
foregoing model did not regard memory $\mathrm{T}$ and $\mathrm{B}$ cells. Hence, the mechanisms by which these memory effects including due to vaccination influence the immune response to SARS-CoV-2 infection and long COVID remain unknown and further investigations including longitudinal observations on prognosis and immune response of unvaccinated and vaccinated patients are essential.

\section{Materials and Methods}

\section{Mathematical Model}

A mathematical model comprising ordinary differential equations (ODEs) was used to investigate host immune responses to SARS-CoV-2 infection. The immune response occurred mainly at the infection site (respiratory tract) and the lymph nodes (Fig. 1). In the latter, APC and Th1 activated CD8+ $\mathrm{T}_{0}$ cells which differentiated into CTLs, and APC and Tfh activated $\mathrm{B}_{0}$ cells which differentiated into $\mathrm{pB}$, then it produced Ig. Following are the model ODEs:

\section{Infection sites}

$$
\begin{aligned}
& d[H] / d t=\lambda_{H}-\delta_{H}[H]-\pi_{I}[H][V] /\left(1+\beta_{I}[I g][V]\right), \\
& d[I] / d t=\pi_{I}[H][V] /\left(1+\beta_{I}[I g][V]\right)-\delta_{I}[I]-k_{I}[I]\left[C T L_{R}\right], \\
& d[D C] / d t=\lambda_{D C}-\delta_{D C}[D C]-\pi_{A P C}\left(1+\alpha_{\text {recruit }}[I N F 1]\right)\left(1+\alpha_{A P C}[I g]\right)[D C][V], \\
& d\left[A P C_{R}\right] / d t=\pi_{A P C}\left(1+\alpha_{\text {recruit }}[I N F 1]\right)\left(1+\alpha_{A P C}[I g]\right)[D C][V]-\delta_{A P C_{R}}\left[A P C_{R}\right]-\mu_{A P C}\left[A P C_{R}\right],
\end{aligned}
$$

$$
\begin{aligned}
& d[V] / d t=\pi_{V}[I] /\left(1+\beta_{V}[I N F 1]\right)-\delta_{V}[V]-\pi_{I}[H][V] /\left(1+\beta_{I}[I g][V]\right)-\pi_{A P C}(1+ \\
& \left.\alpha_{\text {recruit }}[I N F 1]\right)\left(1+\alpha_{A P C}[I g]\right)[D C][V]-\gamma_{I g}[I g][V], \\
& d[I F N 1] / d t=\sigma_{I}[I]+\sigma_{A P C}\left[A P C_{R}\right]-\delta_{I F N 1}[I F N 1] .
\end{aligned}
$$

2. Differentiation of naïve CD8+ T cells into CTLs in lymph nodes

$$
\begin{aligned}
& d\left[A P C_{L}\right] / d t=\mu_{A P C}\left[A P C_{R}\right]-\delta_{A P C_{L}}\left[A P C_{L}\right] \\
& d\left[C D 4+T_{o}\right] / d t=\lambda_{C D 4}-\delta_{C D 4}\left[C D 4+T_{o}\right]-\pi_{T h 1}\left(1+\alpha_{T h 1}[I N F 1]\right)\left[A P C_{L}\right]\left[C D 4+T_{o}\right]- \\
& \pi_{T f h}\left(1+\alpha_{T f h}[I N F 1]\right)\left[A P C_{L}\right]\left[C D 4+T_{o}\right] \\
& d[T h 1] / d t=\pi_{T h 1}\left(1+\alpha_{T h 1}[I N F 1]\right)\left[A P C_{L}\right]\left[C D 4+T_{o}\right]-\delta_{T h 1}[T h 1] \\
& d\left[C D 8+T_{o}\right] / d t=\lambda_{C D 8}-\delta_{C D 8}\left[C D 8+T_{o}\right]-\pi_{C T L}\left[A P C_{L}\right][T h 1]\left[C D 8+T_{o}\right] \\
& d\left[C T L_{L}\right] / d t=\pi_{C T L}\left[A P C_{L}\right][T h 1]\left[C D 8+T_{o}\right]-\delta_{C T L}\left[C T L_{L}\right]-\mu_{C T L}\left(1+\alpha_{\text {recruit }}[I N F 1]\right)\left[C T L_{L}\right] \\
& d\left[C T L_{R}\right] / d t=\mu_{C T L}\left(1+\alpha_{\text {recruit }}[I N F 1]\right)\left[C T L_{L}\right]-\delta_{C T L}\left[C T L_{R}\right]
\end{aligned}
$$

3. Ig production by $\mathrm{pB}$ in lymph nodes

$$
d[T f h] / d t=\pi_{T f h}\left(1+\alpha_{T f h}[I N F 1]\right)\left[A P C_{L}\right]\left[C D 4+T_{o}\right]-\delta_{T f h}[T f h],
$$




$$
\begin{aligned}
& d\left[B_{o}\right] / d t=\lambda_{B}-\delta_{B}\left[B_{o}\right]-\pi_{p B}\left[A P C_{L}\right][T f h]\left[B_{o}\right], \\
& d[p B] / d t=\pi_{p B}\left[A P C_{L}\right][T f h]\left[B_{o}\right]-\delta_{p B}[p B], \\
& d[I g] / d t=\pi_{I g}[p B]-\delta_{I g}[I g]-\xi_{I g}[\operatorname{Ig}][V],
\end{aligned}
$$

The effects considered by the model are summarized below. The rate of infection of the target epithelial cells with free virus is proportional to $\left(1+\beta_{I}[I g][V]\right)^{-1}$ (Eq. 2). Thus, the infection is suppressed by Ig binding the virus. DCs recruited and activated by IFN1 ${ }^{42}$ efficiently capture antibody-neutralized virus ${ }^{43}$ and transform into $\mathrm{APC}_{\mathrm{R}}$. Therefore, the rate of $\mathrm{DC}$ transformation is proportional to $\left(1+\alpha_{\text {recruit }}[I N F 1]\right)\left(1+\alpha_{A P C}[I g]\right)$ (Eq. 4). Viral replication is inhibited by IFN1-induced genes ${ }^{12,44}$. Hence, the viral replication rate is proportional to the inverse of $(1+$ $\left.\beta_{V}[I N F 1]\right)$ and virus neutralized by $\operatorname{Ig}$ is removed at a rate proportional to $\gamma_{I g}[I g]$ (Eq. 5). Induction of the development of CD4+ $\mathrm{T}_{0}$ into Th1 and Tfh by $\mathrm{APC}_{\mathrm{L}}{ }^{45}$ is stimulated by IFN1 ${ }^{46,47}$. In Eq. 12, it is assumed that $\mathrm{CTL}_{\mathrm{L}}$ are recruited and activated by IFN1 ${ }^{46}$ and that the cytolytic activity of CTL $\mathrm{R}_{\mathrm{R}}$ is enhanced ${ }^{46}$.

\section{Simulations}

The ordinary differential equations (1-16) used in the mathematical model of the immune response to SARS-CoV-2 were solved with the COPASI biochemical system simulator (v. 4.28) ${ }^{29}$ to obtain the variable and flux time courses. The initial concentrations and model parameters used in the simulations are listed in the supplementary tables. Several baseline model parameters listed in Tables S1 and S2 were determined such that the baseline model simulation reproduced the time courses for the clinically observed viral load (Fig. 1a) and [Ig] (Fig. 1b). Hence, the baseline simulation was consistent with the DC level 7 mo after infection (Fig. 3). The steady-state solution and sensitivity analysis were also determined with COPASI ${ }^{29}$.

\section{References}

1. Zhou, P. et al. A pneumonia outbreak associated with a new coronavirus of probable bat origin. Nature 579, 270-273 (2020).

2. Chan, J. F.-W. et al. A familial cluster of pneumonia associated with the 2019 novel coronavirus indicating person-to-person transmission: a study of a family cluster. Lancet $\mathbf{3 9 5}$, 514-523 (2020).

3. Huang, C. et al. Clinical features of patients infected with 2019 novel coronavirus in Wuhan, China. Lancet 395, 497-506 (2020).

4. Gupta, A. et al. Extrapulmonary manifestations of COVID-19. Nat Med 26, 1017-1032 (2020).

5. Schultze, J. L. \& Aschenbrenner, A. C. COVID-19 and the human innate immune system. Cell 
184, 1671-1692 (2021).

6. Du, S. Q. \& Yuan, W. Mathematical modeling of interaction between innate and adaptive immune responses in COVID-19 and implications for viral pathogenesis. J Med Virol 92, 1615-1628 (2020).

7. Hernandez-Vargas, E. A. \& Velasco-Hernandez, J. X. In-host Mathematical Modelling of COVID-19 in Humans. Annu Rev Control 50, 448-456 (2020).

8. Kim, K. S. et al. A quantitative model used to compare within-host SARS-CoV-2, MERS-CoV, and SARS-CoV dynamics provides insights into the pathogenesis and treatment of SARS-CoV-2. PLoS Biol 19, e3001128 (2021).

9. Ejima, K. et al. Estimation of the incubation period of COVID-19 using viral load data. Epidemics 35, 100454 (2021).

10. Ghosh, I. Within Host Dynamics of SARS-CoV-2 in Humans - Modeling Immune Responses and Antiviral Treatments. SN Comput Sci 2, 482 (2021).

11. Voutouri, C. et al. In silico dynamics of COVID-19 phenotypes for optimizing clinical management. Proc. Natl. Acad. Sci. U.S.A. 118, (2021).

12. Sa Ribero, M., Jouvenet, N., Dreux, M. \& Nisole, S. Interplay between SARS-CoV-2 and the type I interferon response. PLoS Pathog 16, e1008737 (2020).

13. Bastard, P. et al. Autoantibodies neutralizing type I IFNs are present in $\sim 4 \%$ of uninfected individuals over 70 years old and account for $20 \%$ of COVID-19 deaths. Sci Immunol 6, (2021).

14. Agrawal, A. \& Gupta, S. Impact of aging on dendritic cell functions in humans. Ageing Res Rev 10, 336-345 (2011).

15. Agrawal, A. et al. Altered innate immune functioning of dendritic cells in elderly humans: a role of phosphoinositide 3-kinase-signaling pathway. J Immunol 178, 6912-6922 (2007).

16. Sridharan, A. et al. Age-associated impaired plasmacytoid dendritic cell functions lead to decreased CD4 and CD8 T cell immunity. Age (Dordr) 33, 363-376 (2011).

17. Miyazawa, M. Immunopathogenesis of SARS-CoV-2-induced pneumonia: lessons from influenza virus infection. Inflamm Regen 40, 39-13 (2020).

18. Hoffmann, M. et al. SARS-CoV-2 Cell Entry Depends on ACE2 and TMPRSS2 and Is Blocked by a Clinically Proven Protease Inhibitor. Cell 181, 271-280.e8 (2020).

19. Hamming, I. et al. Tissue distribution of ACE2 protein, the functional receptor for SARS coronavirus. A first step in understanding SARS pathogenesis. J Pathol 203, 631-637 (2004).

20. Paniz-Mondolfi, A. et al. Central nervous system involvement by severe acute respiratory syndrome coronavirus-2 (SARS-CoV-2). J Med Virol 92, 699-702 (2020).

21. Puelles, V. G. et al. Multiorgan and Renal Tropism of SARS-CoV-2. N Engl J Med 383, 590- 
592 (2020).

22. Qi, F., Qian, S., Zhang, S. \& Zhang, Z. Single cell RNA sequencing of 13 human tissues identify cell types and receptors of human coronaviruses. Biochem Biophys Res Commun 526, $135-140$ (2020).

23. Proal, A. D. \& VanElzakker, M. B. Long COVID or Post-acute Sequelae of COVID-19 (PASC): An Overview of Biological Factors That May Contribute to Persistent Symptoms. Front Microbiol 12, 698169 (2021).

24. Pérez-Gómez, A. et al. Dendritic cell deficiencies persist seven months after SARS-CoV-2 infection. Cell Mol Immunol 18, 2128-2139 (2021).

25. Young, B. E. et al. Epidemiologic Features and Clinical Course of Patients Infected With SARS-CoV-2 in Singapore. JAMA 323, 1488-1494 (2020).

26. Yin, S. et al. Longitudinal anti-SARS-CoV-2 antibody profile and neutralization activity of a COVID-19 patient. J Infect 81, e31-e32 (2020).

27. Sette, A. \& Crotty, S. Adaptive immunity to SARS-CoV-2 and COVID-19. Cell 184, 861-880 (2021).

28. Westera, L. et al. Lymphocyte maintenance during healthy aging requires no substantial alterations in cellular turnover. Aging Cell 14, 219-227 (2015).

29. Bergmann, F. T. et al. COPASI and its applications in biotechnology. J Biotechnol 261, 215220 (2017).

30. Chertow, D. et al. SARS-CoV-2 infection and persistence throughout the human body and brain. (2021). doi:10.21203/rs.3.rs-1139035/v1

31. Logue, J. K. et al. Sequelae in Adults at 6 Months After COVID-19 Infection. JAMA Netw Open 4, e210830-e210830 (2021).

32. Del Rio, C., Collins, L. F. \& Malani, P. Long-term Health Consequences of COVID-19. JAMA 324, 1723-1724 (2020).

33. Liotti, F. M. et al. Assessment of SARS-CoV-2 RNA Test Results Among Patients Who Recovered From COVID-19 With Prior Negative Results. JAMA Intern Med 181, 702-704 (2021).

34. Sun, J. et al. Prolonged Persistence of SARS-CoV-2 RNA in Body Fluids. Emerg Infect Dis 26, 1834-1838 (2020).

35. Vibholm, L. K. et al. SARS-CoV-2 persistence is associated with antigen-specific CD8 T-cell responses. EBioMedicine 64, 103230 (2021).

36. Doi, T. et al. Measles virus induces persistent infection by autoregulation of viral replication. Sci. Rep. 6, 37163-9 (2016).

37. Randall, R. E. \& Griffin, D. E. Within host RNA virus persistence: mechanisms and 
consequences. Curr Opin Virol 23, 35-42 (2017).

38. Ireland, D. D. C. et al. Long-term persistence of infectious Zika virus: Inflammation and behavioral sequela in mice. PLoS Pathog 16, e1008689 (2020).

39. KRISTENSSON, K. \& NORRBY, E. Persistence of Rna Viruses in the Central-Nervous-System. Annu Rev Microbiol 40, 159-184 (1986).

40. Rehermann, B. Hepatitis $\mathrm{C}$ virus versus innate and adaptive immune responses: a tale of coevolution and coexistence. J Clin Invest 119, 1745-1754 (2009).

41. Patra, T., Ray, R. B. \& Ray, R. Strategies to Circumvent Host Innate Immune Response by Hepatitis C Virus. Cells 8, 274 (2019).

42. Fitzgerald-Bocarsly, P. \& Feng, D. The role of type I interferon production by dendritic cells in host defense. Biochimie 89, 843-855 (2007).

43. van Montfort, T., Nabatov, A. A., Geijtenbeek, T. B. H., Pollakis, G. \& Paxton, W. A. Efficient capture of antibody neutralized HIV-1 by cells expressing DC-SIGN and transfer to CD4+ T lymphocytes. J Immunol 178, 3177-3185 (2007).

44. Makris, S., Paulsen, M. \& Johansson, C. Type I Interferons as Regulators of Lung Inflammation. Front Immunol 8, 259 (2017).

45. Swain, S. L., McKinstry, K. K. \& Strutt, T. M. Expanding roles for CD4 ${ }^{+}$T cells in immunity to viruses. Nat Rev Immunol 12, 136-148 (2012).

46. Huber, J. P. \& Farrar, J. D. Regulation of effector and memory T-cell functions by type I interferon. Immunology 132, 466-474 (2011).

47. Cucak, H., Yrlid, U., Reizis, B., Kalinke, U. \& Johansson-Lindbom, B. Type I interferon signaling in dendritic cells stimulates the development of lymph-node-resident $\mathrm{T}$ follicular helper cells. Immunity 31, 491-501 (2009).

\section{Acknowledgements}

This work was partly supported by JSPS KAKENHI Grant No. JP20K05431.

\section{Author Contributions}

T.S. developed the mathematical model, performed the simulations, and analyzed the computational data. T.S. and K.H. discussed the results and wrote the paper.

\section{Competing interests}

The authors declare no competing interests. 


\section{Data availability}

The COPASI input data used to generate the data for the current study are available from the corresponding author (TS) upon reasonable request.

\section{Additional information}

Supplementary information is available at 


\section{Supplementary Files}

This is a list of supplementary files associated with this preprint. Click to download.

- SllmmuneResponsesSARSCoV2.pdf 